\title{
Antiplatelet use in practice
}

Amie Bain, Pharmacist, Academic Practitioner and Senior Lecturer in Therapeutics, School of Applied Sciences, University of Huddersfield, Queensgate. Email: a.bain@hud.ac.uk

\begin{abstract}
Antiplatelets are widely used drugs that can prevent platelet activation and subsequent aggregation, inhibiting arterial thrombus formation that can contribute to the development of myocardial infarction and stroke. The use of antiplatelets for secondary prevention of cardiovascular disease is supported by a strong and compelling evidence base, with rigorous clinical trials supporting the use of varying combinations of antiplatelets for different indications. A sound understanding of how antiplatelets work is needed to promote their safe and effective use. This article briefly describes the process of platelet activation, aggregation and subsequent thrombus formation, and will discuss the mechanism of action of antiplatelets and their place in therapy.
\end{abstract}

Key words Antiplatelet drugs, Platelets, Aspirin, Pharmacology, Prescribing

\section{Introduction}

Antiplatelet drugs are widely used for a number of indications, mainly involving the prevention of arteriothrombotic events. Most patients with established cardiovascular disease who are not already receiving anticoagulation therapy will be prescribed at least one antiplatelet drug, often for longterm use. The evidence for antiplatelet use in reducing cardiovascular complications in established disease is compelling, and their use is strongly recommended by both national and international bodies for patients with established cardiovascular disease. A sound understanding of both how antiplatelets work and the evidence for their indicated uses can help practitioners improve patient knowledge, acceptance and adherence to this important class of medication. The current article briefly describes the process of platelet activation, aggregation and subsequent thrombus formation that results in cardiovascular events, and discusses the mechanism of action of antiplatelets, as well as their place in therapy.

\section{Role of platelets}

Platelets are essential components of haemostasis and pathological thrombus formation, and work to maintain the integrity of the circulation (Kroll, 1998). Following vessel injury, the vascular endothelium is breached, exposing subendothelial proteins such as von Willebrand factor (vWF) and collagen to blood (Gale, 2011). These proteins stick to glycoprotein receptors (e.g. Ib and VI) which are expressed on the surface of platelets. As a result, platelets adhere both to the vessel at the site of injury and to each other, forming a platelet plug (Golebiewska and Poole, 2015).

This adhesion then leads to platelet activation as well as the formation and upregulation of platelet glycoprotein Ilb/Illa receptor complexes; the platelets change shape and release pro-aggregating factors (e.g. thromboxane A2, which is synthesised from arachidonic acid by COX-1), contributing to further aggregation. Fibrinogen in the plasma then crosslinks glycoprotein IIb/IIla receptors on the platelets, causing irreversible aggregation (Figure 1). 
Various physiological mechanisms regulate this process, including endothelium-derived prostacyclin (prostaglandin 12/PGI2) and nitric oxide, which inhibit platelet adhesion and activation (Waller and Sampson, 2017). This normal physiological process of platelet adhesion and activation can aid processes such as wound healing and the repair of ruptured atherosclerotic plaques. However, when it is uncontrolled-which may happen in the presence of vessel disease such as atherosclerosis and endothelial damage - it can lead to thrombus formation, vessel occlusion and ischaemia/infarction.

To reduce the risk of unwanted pathology, it is sometimes necessary to use antiplatelet drugs to interfere with the platelet aggregation process. Antiplatelets decrease platelet aggregation and/or subsequent activation, and inhibit thrombus formation-particularly in the arterial circulation where thrombi are mainly composed of platelets, as opposed to the venous circulation where thrombi are mainly composed of fibrin (Previtali et al, 2011).

There are a variety of drugs in this class, with individual agents targeting different parts of the platelet aggregation process to reduce the risk of thrombus formation. Examples of how different antiplatelet agents work are given in the next section.

\section{Mechanism of action}

Antiplatelet drugs can either block pro-aggregation and activation mechanisms, or mimic endogenous inhibitory mechanisms. For example, the glycoprotein Ilb/Illa receptor complex is the final common step in the processes of thrombus formation, whereby conformational changes allow for cross-linking via fibrinogen. By interfering with the formation, upregulation and function of this receptor complex, platelet aggregation can be limited.

Thromboxane A2 (TXA2) is a pro-aggregation mediator that activates platelets and upregulates glycoprotein Ilb/Illa receptors. Inhibiting the synthesis and action of TXA2 will therefore inhibit platelet activation and aggregation. The most commonly used antiplatelet-aspirin-may exert its action by inhibiting COX-1, the enzyme that catalyses production of TXA2 from arachidonic acid. Because platelets have no nucleus, they are unable to regenerate once damaged. The antiplatelet effect therefore lasts the lifetime of the platelet (around 10 days), and is complete when $75-150 \mathrm{mg}$ is given once daily. Adherence to daily aspirin treatment is important, as regularly missing doses will compromise its antithrombotic protection.

Adenosine diphosphate (ADP) is another pro-aggregating factor that contributes to platelet activation, and glycoprotein Ilb/IIla receptor formation and upregulation. Drugs that block ADP receptors on platelets (e.g. P2Y12 receptors) such as clopidogrel, prasugrel, ticagrelor and cangrelor, can therefore inhibit platelet aggregation and activation. Ticagrelor and prasugrel have a more rapid onset of action and less interpatient variability than clopidogrel (Jernberg et al, 2006; Wallentin et al, 2009), but have fewer licensed indications.

Glycoprotein Ilb/Illa receptors can also be downregulated by agents that mimic endogenous inhibitory mediators such as PGI2; dipyridamole and epoprostenol, for example, increase the concentration of platelet cAMP (dipyridamole inhibits the reuptake of adenosine, which stimulates production of intracellular adenylyl cyclase) - an endogenous cyclic nucleotide that inhibits the activation of glycoprotein IIb/IIla receptors on the platelet (Waller and Sampson, 2017: 171-173). 
Abciximab, tirofiban and eptifibatide are examples of glycoprotein Ilb/Illa receptor antagonists that act on the final step of platelet aggregation: they bind to the receptor complex, blocking fibrinogen from attaching and binding platelets together (Rang et al, 2012). Abciximab is a monoclonal antibody, whereas tirofiban and eptifibatide are small molecule glycoprotein inhibitors which have a quicker offset of action.

Because multiple signalling pathways are involved in platelet activation and aggregation, antiplatelets that target different elements of the platelet aggregation process are often used in combination to maximise the overall antiplatelet effect (Table 1). In practice, this almost always consists of using aspirin in combination with another antiplatelet (e.g. a P2Y12 inhibitor); this is often referred to as dualantiplatelet therapy (DAPT). The need for combination therapy will depend on the patient and the indication(s), but general recommendations based on national guidance are outlined below.

\section{Indications}

The end result of uncontrolled platelet aggregation is the formation of a blood clot-a thrombus. Thrombi can occlude blood vessels, causing blood flow to be obstructed and consequential ischaemia to tissue (Golebiewska and Poole, 2015). When pieces of thrombi become detached and travel through the circulation, lodging somewhere else to cause an obstruction, it is known as an embolus (hence the term thromboembolic disease). As platelet-rich thrombi are typically found in arteries, antiplatelet agents are mostly used to treat conditions that arise as a result of blood clots in the arterial circulation.

Acute coronary syndrome (ACS), for example, occurs as a result of thrombus formation in the coronary arteries, leading to ischaemic damage. Long-term antiplatelet therapy is recommended in all patients after an episode of ACS, including ST-elevated myocardial infarction (STEMI), non-ST elevation myocardial infarction (NSTEMI) and unstable angina, to prevent further platelet aggregation and thrombus formation (National Institute for Health and Care Excellence (NICE), 2013a; 2013b). In practice, this is often DAPT consisting of long-term aspirin $75 \mathrm{mg}$ daily, with the addition of a P2Y12 antagonist (e.g. ticagrelor or clopidogrel) for 12 months (Table 2). The use of DAPT allows for the prevention of further thrombus formation at the site of endothelial damage, as well as supporting endothelialisation in patients who have had a stent fitted. In addition, P2Y12 inhibitor therapy helps to reduce the likelihood of stent thrombosis in patients who undergo coronary artery stent implantation, a rare but potentially fatal complication of percutaneous coronary intervention (PCI).

Other examples of arterial thromboembolic disease include ischaemic stroke and transient ischaemic attack (TIA). Here, thrombi obstruct blood flow in arteries in the brain (e.g. the middle cerebral artery) with subsequent ischaemia. Patients who have experienced an ischaemic stroke or a TIA will routinely be offered long-term antiplatelet therapy to reduce the risk of further platelet aggregation and repeat episodes (Intercollegiate Stroke Working Party, 2016). The exception to this is if atrial fibrillation (AF) is also present, and there is no other indication for DAPT. In this case, after the acute phase, an anticoagulant (e.g. warfarin, apixaban, rivaroxaban, dabigatran or edoxaban) will be offered instead of an antiplatelet because they are significantly more effective in patients with AF (Aguilar et al, 2007). If both DAPT and anticoagulation are indicated (e.g. if a patient has a history of AF-related stroke and recent myocardial infarction (MI)) and used concomitantly, the duration of 
DAPT treatment is usually shortened to mitigate against bleeding risk as assessed using the HASBLED risk score.

Although antiplatelets are recommended for secondary prevention of thromboembolic events in people with established ischaemic cardiovascular or cerebrovascular disease, they are not generally recommended for primary prevention as the risk of bleeding outweighs therapeutic benefit (Perk et al, 2012; Scottish Intercollegiate Guidelines Network (SIGN), 2013). For individuals at risk (e.g. in those with hypertension and renal impairment), expert opinion varies, with weak evidence leading some clinicians to prescribe aspirin $75 \mathrm{mg}$ daily for primary prevention (Nansseu and Noubiap, 2015), despite its unlicensed indication.

Antiplatelets can also be used during or following angioplasty/PCl with stenting and coronary artery bypass grafts (CABG) to prevent reocclusion or restenosis of the affected vessel(s). They may also be used for peripheral arterial disease, including carotid artery disease and peripheral artery disease (Table 2).

\section{The evidence}

The value of antiplatelets for secondary prevention of cardiovascular and cerebrovascular disease is well-established. For patients at high risk, taking long-term antiplatelet therapy has been shown to reduce the combined outcome of non-fatal $\mathrm{MI}$, non-fatal stroke or vascular death by about $25 \%$ (Antithrombotic Trialists' Collaboration, 2002). For ACS, various trials have proven the benefit of antiplatelet therapy for secondary prevention. Aspirin is the oldest and most widely used antiplatelet, with the ISIS-2 trial (ISIS-2 (Second International Study of Infarct Survival) Collaborative Group, 1988) demonstrating its benefit regarding short- and long-term mortality (both vascular and all-cause), and morbidity (i.e. reinfarction). When clopidogrel is added to aspirin, there is a further reduction in cardiovascular mortality, non-fatal $\mathrm{MI}$ and stroke, as demonstrated in the CURE and COMMIT trials (Yusuf et al, 2001; Chen et al, 2005), although this increases major and minor bleeding risk.

The TRITON-TIMI-38 trial (Wiviott et al, 2007) demonstrated the benefits of prasugrel over clopidogrel with respect to reduction in cardiovascular morbidity and mortality (although it increased bleeding) in the event of ACS scheduled for PCI. The TRILOGY-ACS trial (Roe et al, 2012) did not find the same benefits in patients with ACS who were not scheduled for revascularisation. More recently, the PLATO trial demonstrated a significant reduction in composite death from vascular causes, MI or stroke with no difference in major or severe bleeding when ticagrelor was used instead of clopidogrel (Wallentin et al, 2009). This trial directed the European Society of Cardiology (ESC) to recommended ticagrelor as the P2Y12 inhibitor of choice in combination with aspirin for ACS in its guidelines (Roffi et al, 2016).

For stable angina, aspirin is the most cost-effective antiplatelet. The CAPRIE trial (CAPRIE Steering Committee, 1996) showed that clopidogrel is as effective as aspirin in reducing the risk of vascular events ( $\mathrm{Ml}$, stroke and vascular death), so can be used in patients who cannot tolerate aspirin. Subgroup analyses have demonstrated the benefit of clopidogrel over aspirin in both peripheral arterial disease and multivessel disease, making it the drug of choice for these indications (NICE, 2018).

In the event of ischaemic stroke, the use of aspirin was shown to reduce mortality and improve functional recovery by $2.7 \%$ when given within 48 hours of symptom onset (after haemorrhagic 
stroke has been ruled out) in the CAST and IST trials (Chen et al, 2000). When used in the long term, aspirin can reduce the likelihood of stroke recurrence by up to $23 \%$. Clopidogrel can reduce this risk by a further $8 \%$ when used alone, but increases the risk of life-threatening bleeding when used with aspirin (Walker and Whittlesea, 2012: 153). Aspirin and dipyridamole are currently recommended by NICE for TIA because clopidogrel is not licensed for TIA (NICE, 2010). However, this combination is no better than clopidogrel monotherapy (Sacco et al, 2008), which is recommended in both stroke and TIA by the Intercollegiate Stroke Working Party (2016). Current recommendations for the above indications are summarised in Table 2.

\section{Side-effects and interactions}

Most antiplatelets are associated with an increased risk of haemorrhage, which is greater at higher maintenance doses (e.g. aspirin after CABG or to treat pain in certain circumstances), when antiplatelets are used in combination or in situations necessitating the use of an antiplatelet with an anticoagulant (e.g. aspirin and warfarin). For patients, this might mean an increased propensity to bruise easily, and cuts and grazes may take longer to stop bleeding than usual; however, unless bleeding is profuse and/or protracted, this is not usually a cause for concern.

Aspirin is associated with a high incidence of gastrointestinal side-effects. This is because it inhibits COX -1 , which prevents the production of prostaglandins, leading to a reduction in the secretion of mucus that would otherwise coat the lining of the stomach to protect it from acidic contents. Inhibition therefore depletes the protective layer and exposes the stomach lining to the acidic content therein, causing dyspepsia-like symptoms. It is recommended that aspirin should be taken with or after food to minimise this effect (Lanas and Scheiman, 2007). In some individuals at risk of these complications (e.g. those with a history of dyspepsia), a proton pump inhibitor (PPI) (e.g. lansoprazole) can be prescribed concomitantly, or clopidogrel could be used instead. Gastrointestinal complications are not reduced when enteric coated aspirin is used (Kelly et al, 1996), so it is not routinely prescribed.

Clopidogrel is metabolised by the CYP2C19 enzyme, giving rise to potential interactions with some other drugs such as omeprazole, fluconazole and carbamazepine, which may compromise its antiplatelet activity. Ticagrelor is a CYP3A4 inhibitor so should be avoided (or used very cautiously) with concomitant clarithromycin, digoxin or simvastatin. Increased monitoring, reviewed doses or alternative agents may be recommended for these cases.

\section{Contraindications and cautions}

The use of antiplatelets in the presence of pathological bleeding (e.g. peptic ulcer or intracranial haemorrhage) or haemorrhagic disorders (e.g. thrombocytopenia) is contraindicated. Antiplatelets should be discontinued around a week before certain surgical or invasive diagnostic procedures if an antiplatelet effect is not desirable, and should be prescribed alongside other agents that predispose to bleeding (e.g. NSAIDS and SSRIs) with caution.

Prasugrel should not be given to patients who have had a stroke or TIA because of the increased risk of fatal bleeding, and requires dose adjustment based on age and weight (Table 2). Because dipyridamole has vasodilator properties, it can worsen migraine symptoms, and should also be used with caution in severe coronary heart disease, heart failure and myasthenia gravis. Patients who have respiratory disease should be given aspirin or P2Y12 inhibitors with caution, because they have the side-effects of bronchospasm and dyspnoea respectively (although this does not usually lead to 
discontinuation). To manage these potential interactions, monitoring may be increased, and alternative agents offered if necessary.

\section{Conclusion}

The use of antiplatelet drugs, alone or in combination, is widespread among patients with cardiovascular disease. Evidence for the use of antiplatelets in secondary prevention is substantial, and they are generally safe and effective. Practitioners need a sound understanding of both how antiplatelets work and the evidence for their indicated uses to improve patient knowledge, acceptance and adherence to this class of drug.

\section{Key Points}

Antiplatelet drugs are widely used to prevent complications in established cardiovascular disease.

A variety of antiplatelet drugs are available; they target different parts of the platelet aggregation process to reduce the risk of thrombus formation.

Common indications for antiplatelet therapy include secondary prevention of acute coronary syndrome and non-AF-related ischaemic stroke, as well as peripheral arterial disease and stable angina.

Although commonly used, antiplatelet therapy is not without risk. Interacting medications, sideeffects, bleeding risk and past medical history should all be considered when using antiplatelet agents in practice

\section{CPD Reflection Questions}

How would you describe the action of antiplatelets to patients in your setting?

What factors need to be considered before prescribing antiplatelets to patients?

What information is important to communicate to patients in order to promote the safe and effective use of antiplatelets?

\section{References}

Aguilar MI, Hart R, Pearce LA. Oral anticoagulants versus antiplatelet therapy for preventing stroke in patients with non-valvular atrial fibrillation and no history of stroke or transient ischemic attacks. Cochrane Database Syst Rev. 2007; (3):CD006186.

https://doi.org/10.1002/14651858.CD006186.pub2

Al-Attar, N. Aspirin therapy after coronary surgery. EJ Cardiol Pract. 2009; 8(9)

Al Jaaly E, Zakkar M, Pufulete M, Ciulli F, Angelini GD. Dual antiplatelet therapy after coronary artery bypass grafting: do we have a consensus? J Integr Cardiol. 2015; 1(4).

https://doi.org/10.15761/JIC.1000126 
Antithrombotic Trialists' Collaboration. Collaborative meta-analysis of randomised trials of antiplatelet therapy for prevention of death, myocardial infarction, and stroke in high risk patients. BMJ. 2002; 324(7329):71-86

CAPRIE Steering Committee. A randomised, blinded, trial of clopidogrel versus aspirin in patients at risk of ischaemic events (CAPRIE). CAPRIE Steering Committee. Lancet. 1996; 348(9038):1329-1339

Chen ZM, Sandercock P, Pan HC et al. Indications for early aspirin use in acute ischemic stroke : A combined analysis of 40000 randomized patients from the chinese acute stroke trial and the international stroke trial. On behalf of the CAST and IST collaborative groups. Stroke. 2000; 31(6):1240- 1249

Chen ZM, Jiang LX, Chen YP et al. Addition of clopidogrel to aspirin in 45,852 patients with acute myocardial infarction: randomised placebocontrolled trial. Lancet. 2005; 366(9497):1607-1621. https://doi. org/10.1016/S0140-6736(05)67660-X

Eikelboom JW, Hirsh J, Spencer FA, Baglin TP, Weitz JI. Antiplatelet drugs: antithrombotic therapy and prevention of thrombosis, 9th ed: American College of Chest Physicians evidence-based clinical practice guidelines. Chest. 2012; 141(2 Suppl):e89S-e119S. https://doi.org/10.1378/chest.112293

Gale AJ. Continuing education course \#2: current understanding of hemostasis. Toxicol Pathol. 2011; 39(1):273-280. https://doi. org/10.1177/0192623310389474

Golebiewska EM, Poole AW. Platelet secretion: from haemostasis to wound healing and beyond. Blood Rev. 2015; 29(3):153-162. https://doi. org/10.1016/j.blre.2014.10.003

Intercollegiate Stroke Working Party. National clinical guideline for stroke. London: Royal College of Physicians; 2016. https://www.strokeaudit.org/ Guideline/Full-Guideline.aspx (accessed 8 May 2018)

ISIS-2 (Second International Study of Infarct Survival) Collaborative Group. Randomised trial of intravenous streptokinase, oral aspirin, both, or neither among 17,187 cases of suspected acute myocardial infarction: ISIS- 2. ISIS-2 (Second International Study of Infarct Survival) Collaborative Group. Lancet. 1988; 2(8607):349-360

Jernberg T, Payne CD, Winters KJ et al. Prasugrel achieves greater inhibition of platelet aggregation and a lower rate of non-responders compared with clopidogrel in aspirin-treated patients with stable coronary artery disease. Eur Heart J. 2006; 27(10):1166-1173. https://doi.org/10.1093/eurheartj/ ehi877

British National Formulary. 2018. https://bnf.nice.org.uk (accessed 8 May)

Kelly JP, Kaufman DW, Jurgelon JM, Sheehan J, Koff RS, Shapiro S. Risk of aspirin-associated major upper-gastrointestinal bleeding with entericcoated or buffered product. Lancet. 1996;

348(9039):1413-1416. https:// doi.org/10.1016/S0140-6736(96)01254-8

Kirchhof P, Benussi S, Kotecha D et al. 2016 ESC Guidelines for the management of atrial fibrillation developed in collaboration with EACTS. Eur J Cardiothorac Surg. 50(5): e1-e88

Kroll MH, Sullivan R. Mechanisms of platelet activation. In: Loscalzo J, Schafer Al, eds. Thrombosis and hemorrhage. Baltimore: Lippincott William \& Wilkins; 1998: 261-91 
Lanas A, Scheiman J. Low-dose aspirin and upper gastrointestinal damage: epidemiology, prevention and treatment. Curr Med Res Opin. 2007; 23(1):163-173.

https://doi.org/10.1185/030079907X162656

Nansseu JR, Noubiap JJ. Aspirin for primary prevention of cardiovascular disease. Thromb J. 2015; 13: 38. https://doi.org/10.1186/s12959-015-0068-7

National Institute for Health and Care Excellence. Guidance on the use of glycoprotein Ilb/IIla inhibitors in the treatment of acute coronary syndromes (technology appraisal guidance 47). 2002. London: NICE. https://www.nice.org.uk/ta47 (accessed 8 May 2018)

National Institute for Health and Care Excellence. Stroke and transient ischaemic attack in over 16s: diagnosis and initial management. Clinical guideline [CG 68]. 2008. London: NICE. https://www.nice.org.uk/cg68 (accessed 8 May 2018)

National Institute for Health and Care Excellence. Clopidogrel and modifiedrelease dipyridamole for the prevention of occlusive vascular events. 2010. Technology appraisal guidance [TA210]. London: NICE. https://www.nice. org.uk/ta210 (accessed 8 May 2018)

National Institute for Health and Care Excellence. Unstable angina and NSTEMI: early management. Clinical guideline [CG94]. 2013a. London: NICE. www.nice.org.uk/cg94 (accessed 8 May 2018)

National Institute for Health and Care Excellence. Myocardial infarction: cardiac rehabilitation and prevention of further cardiovascular disease. Clinical guideline [CG172]. 2013b. London: NICE. www.nice.org.uk/cg172 (accessed 8 May 2018)

National Institute for Health and Care Excellence. Peripheral arterial disease: diagnosis and management. Clinical guideline [CG147]. 2018. London: NICE. www.nice.org.uk/cg147 (accessed 8 May 2018)

Perk J, De Backer G, Gohlke H et al. European Guidelines on cardiovascular disease prevention in clinical practice (version 2012). The Fifth Joint Task Force of the European Society of Cardiology and Other Societies on Cardiovascular Disease Prevention in Clinical Practice (constituted by representatives of nine societies and by invited experts). Eur Heart J. 2012; 33(13):1635-1701. doi: 10.1093/eurheartj/ehs092

Previtali E, Bucciarelli P, Passamonti SM, Martinelli I. Risk factors for venous and arterial thrombosis. Blood Transfus. 2011; 9(2):120-138. https://doi. org/10.2450/2010.0066-10

Rang H, Ritter J, Flower R, Henderson G, Dale M. Rang and Dale's pharmacology. 2016. Edinburgh: Churchill Livingstone/Elsevier; 2016: 318-330

Roe MT, Armstrong PW, Fox KA et al. Prasugrel versus clopidogrel for acute coronary syndromes without revascularization. N Engl J Med. 2012; 367(14):1297-1309.

https://doi.org/10.1056/NEJMoa1205512

Roffi M, Patrono C, Collet JP et al. 2015 ESC Guidelines for the management of acute coronary syndromes in patients presenting without persistent ST- segment elevation: Task Force for the Management of Acute Coronary Syndromes in Patients Presenting without Persistent ST-Segment Elevation of the European Society of Cardiology (ESC). Eur Heart J. 2016; 37(3):267-315.

https://doi.org/10.1093/eurheartj/ehv320 
Scottish Intercollegiate Guidelines Network. SIGN 129: Antithrombotics: indications and management. Edinburgh: SIGN. 2013. www.sign.ac.uk/ sign-129-antithrombotics-indications-andmanagement.html (accessed 8 May 2018)

Sacco RL, Diener HC, Yusuf $S$ et al. Aspirin and extended-release dipyridamole versus clopidogrel for recurrent stroke. N Engl J Med. 2008 Sep 18;359(12):1238-1251.

https://doi.org/10.1056/NEJMoa0805002

Valgimigli M, Bueno H, Byrne RA et al. 2017 ESC focused update on dual antiplatelet therapy in coronary artery disease developed in collaboration with EACTS: The Task Force for dual antiplatelet therapy in coronary artery disease of the European Society of Cardiology (ESC) and of the European Association for Cardio-Thoracic Surgery (EACTS). Eur Heart J. 2018; 39(3):213-260.

https://doi.org/10.1093/eurheartj/ehx419

Walker R, Whittlesea C. Clinical pharmacy and therapeutics. 2012. Edinburgh: Churchill Livingstone/Elsevier

Wallentin L, Becker RC, Budaj A et al. Ticagrelor versus clopidogrel in patients with acute coronary syndromes. N Engl J Med. 2009; 361(11):1045-1057. https://doi.org/10.1056/NEJMoa0904327

Waller D, Sampson T. Medical pharmacology and therapeutics. 5th edn. 2017. Edinburgh: Elsevier

Wiviott SD, Braunwald E, McCabe $\mathrm{CH}$ et al. Prasugrel versus clopidogrel in patients with acute coronary syndromes. N Engl J Med. 2007; 357(20):2001-2015.

https://doi.org/10.1056/NEJMoa0706482

Yusuf S, Zhao F, Mehta SR et al. Effects of clopidogrel in addition to aspirin in patients with acute coronary syndromes without ST-segment elevation. N Engl J Med. 2001 Aug 16;345(7):494502.https://doi.org/10.1056/ NEJMoa010746 